\title{
La confianza en el carácter de Dios y la salud mental*
}

\author{
Trust in the character of god and mental health \\ A confiança no caráter de deus e a saúde mental
}

DOI: https://doi.org/10.21803/pensam.v11i21-1.269

Ciro Ernesto Redondo Mendoza

https://orcid.org/0000-0002-2357-7289

Luis Enrique Ribero Suárez

https://orcid.org/0000-0002-5861-2216

\section{¿Cómo citar este artículo?}

Redondo, C. \& Ribero, L. (2018). La confianza en el carácter de Dios y la salud mental. Pensamiento Americano, 11(22), 121-134.

DOI: https://doi.org/10.21803/pensam.v17i21-1.269

\section{Resumen}

Introducción. La "salud mental", conlleva implicaciones epistemológicas en la medida que se adopten posturas que varían desde posiciones positivas (como un "estado de completo bienestar ") o negativas, incluso si se admite, que el concepto es más amplio que la ausencia de trastornos mentales. Objetivo. Identificar la relación que existe entre los niveles de salud mental, y las interpretaciones de creencias sobre el carácter de Dios y su confianza en él, de personas pertenecientes a las comunidades de fundamento cristiano. Metodología. El estudio se realizó con 215 miembros regulares de diferentes comunidades religiosas de fundamento cristiano, fue desarrollado bajo el enfoque cuantitativo, con nivel descriptivo y de alcance transversal. El analisis es llevado al fenomeno de la práctica religiosa o espiritual, ya que para los autores, las personas que pertenecen a comunidades religiosas no están exentas de este panorama. Resultados. Se evidencia alta presencia de salud mental y confianza en Dios. De igual manera se encontró que existe una tendencia a tener una alta confianza en el carácter de Dios independientemente de los años que las personas lleven en la iglesia. Conclusión. Aún existe un gran espectro de interrogantes, posibilidades y retos que se ciernen sobre este estudio, sin embargo, avanza en una línea empírica de descripción de realidades que permiten visibilizar algunas concepciones teóricas de estudios que la preceden.

Palabras Claves: Confianza, Dios, salud mental, iglesia, comunidad religiosa.

\begin{abstract}
"Mental health" carries epistemological implications to the extent that positions are adopted that vary from favourable positions (such as a "state of complete well-being") or negative, even if it is admitted, that the concept is broader than the absence of mental illnesses. The relationship between the levels of mental health, and the interpretations of beliefs about the character of God and his confidence in him, of persons belonging to communities of Christian foundation will be identified. The study was conducted with 215 regular members of different religious communities of Christian foundation, was developed under the quantitative approach, with descriptive level and transversal scope. The analysis is led to the phenomenon of religious or spiritual practice since for the authors, people belonging to religious communities are not exempt from this panorama. Results. There is a high presence of mental health and trust in God.
\end{abstract}

* Artículo original derivado del proyecto de investigación: "La confianza en el carácter de dios y la salud mental" ejecutado entre enero y noviembre de 2017, financiado por la Corporación Universitaria Adventista de Colombia UNAC 
Similarly, it was found there is a tendency to have high confidence in the character of God regardless of the years that people have been in the church. There is still a broad spectrum of questions, possibilities and challenges that loom over this study. However, it advances in an empirical line of description of realities that allow us to make visible some theoretical conceptions of studies that precede it.

Keywords: Trust, God, mental health, church, religious community.

\section{Resumo}

A "saúde mental" leva a implicações epistemológicas na medida em que de adotem posturas que variam de posições positivas (como um "estado completo de bem-estar" ou negativas, inclusive admite-se que o conceito é mais amplo que a ausência de transtornos mentais. Será identificada a relação que existe entre os níveis de saúde mental e as interpretações das crenças sobre o caráter de Deus e sua confiança nele, de pessoas que pertencem às comunidades de fundamento cristão. 0 estudo foi feito com 215 membros regulares de diferentes comunidades religiosas de fundamento cristão, a pesquisa se desenvolveu baixo o enfoque quantitativo, com nível descritivo e de alcance transversal. A análise leva o fenômeno da prática religiosa ou espiritual, já que para os autores, as pessoas que pertencem às comunidades religiosas não estão isentas deste panorama. Resultados: evidencia-se alta presença de saúde mental e confiança em Deus. De igual modo, existe uma tendência a ter uma alta confiança no caráter de Deus independentemente dos anos que as pessoas têm como frequentadoras das igrejas. Existe, ainda, um grande espectro de perguntas, possibilidades e desafios que abrangem o estudo, no entanto, este avança em uma linha empírica de descrição de realidades que permitem visibilizar algumas concepções teóricas de estudos que a precedem.

Palavras chave: Confiança, Deus, saúde mental, Igreja, comunidade religiosa

\section{Perfil}

Doctor en Ciencias de la educación, docente e investigador de la Facultad de Teología en la Corporación Universitaria Adventista de Colombia UNAC y docente del programa de Psicología en la Corporación Universitaria Minuto de Dios UNIMINUTO, E-mail: credondo@unac.edu.co, ciro0980@ hotmail.com

\section{Perfil}

Vicerrector académico, docente e investigador en la Corporación Universitaria Adventista de Colombia UNAC, E-mail: viceacademica@unac. edu.co

\section{Ciro Ernesto Redondo Mendoza}

Doctor en Ciencias de la educación

\section{Luis Enrique Ribero Suárez}

Ph.D en Teología. 


\section{Introducción}

Hablar de salud mental conlleva implicaciones epistemológicas en la medida que se adopten posturas que varían desde posiciones positivas (entendida como "Psicología Salutogénica o Positiva") y que la Organización Mundial de la Salud (OMS), define como "(...) un estado de completo bienestar, en el que una persona es consciente de sus capacidades y puede afrontar las tensiones normales de la vida" ("Hay que invertir", 2011); o negativas (entendida desde la "Psicopatología"), que desde el punto de vista de Oramas Viera, Santana López \& Vergara Barrenechea (2006), implica que al "proceso de la salud mental se accede por el polo negativo, obviamente por la necesidad de dar solución inmediata al problema de la enfermedad, predominando en su abordaje un enfoque médico psicopatológico" (p.34).

Es así como, a partir del enfoque psicopatológico, la OMS (2004) documenta en un número aproximado (y creciente) de 450 millones de personas que se encuentran con un trastorno mental o de la conducta, panorama que no difiere mucho en Colombia donde...

"Dos de cada cinco colombianos han sufrido alguna vez en su vida de un trastorno mental... Solamente el $23 \%$ recibe tratamiento. El resto ha tenido que aprender a convivir con la depresión, los cambios de personalidad, la ansiedad, los ataques de pánico y las alucinaciones", entre otras (Suárez, 2010, p.1).

Esta es una noticia reveladora que se vuelve alarmante si se consideran los resultados de la actual situación que presenta la reportera Mariana Suárez en un artículo del periódico El Espectador, en el cual describe cómo en Colombia

“(..) el $30 \%$ de los trastornos mentales... son fruto de adversidades asociadas con la disfunción familiar. Estamos hablando de divorcios, violencia, la muerte de algún padre, abuso sexual, etc. De acuerdo con la investigación, si logramos controlar estos factores se reducirían hasta en un $23 \%$ los casos de trastornos afectivos, en un $27 \%$ los de abuso y dependencia de las drogas, en un $31 \%$ los de ansiedad y en un $42 \%$ los de conducta" (Suárez, 2010, p. 1).

A pesar que el anterior informe, es solo de carácter informativo, corrobora lo que refiere Suárez (2010). Al admitir que el concepto de salud mental es más amplio que la ausencia de trastornos mentales. Aunque las siguientes estadísticas no presentan la relación entre salud mental y religiosidad, de todas maneras son evidentes los problemas psicopatológicos en la población medellinense, del resto Colombia y de otros países.

El Grupo de Investigación en Salud Mental de la Universidad CES, reportó que en Medellín, de acuerdo con los informes del Sistema General de Seguridad Social en Salud, para 2014, en consulta externa se registraron un total de 162.899 casos asociados a trastornos mentales y del comportamiento, que a su vez equivalieron al 2,18\% del total de las consultas (7'476.763). Los cinco principales diagnósticos son trastorno mixto de ansiedad y depresión, perturbación de la actividad y de la atención, trastorno de ansiedad no especificado, trastorno de ansiedad generalizada y episodio depresivo moderado, que representa el $29.7 \%$ del total de las consultas. A su vez, la Secretaría de Salud identificó un aumento en el número de suicidios. Entre enero y septiembre de 2015, se presentaron 115 muertes confirmadas por este evento en la ciudad, nueve casos más que el total consolidado de 2014 ("Revelan radiografía de la salud", 2016).

Las personas que pertenecen a comunidades

Pensamiento Americano Vol. 11 (22) • 2018 • Julio-Diciembre · Corporación Universitaria Americana · Barranquilla, Colombia • ISSN: $2027-2448$. 
religiosas no están exentas en este panorama; sobre la relación entre religión o espiritualidad y salud mental, hay literatura amplia; Hauck (1985) citado por Cordelia y Kirsten (2010), por ejemplo, expresó que la religión favorece el odio hacia uno mismo, la culpa y los complejos de inferioridad. Sostiene, además, que esta es una fuente muy común de trastornos emocionales como la autoculpabilidad, ya que esta forma de sentirse lleva a perpetuar los mismos problemas o incluso la creación de otros. Esta es una mirada polarizada sobre el asunto de la religiosidad en la vida de los individuos pertenencientes a alguna denominacion religiosa.

Otros autores como Gromm (1994) citado por Ramos (2006), afirman que a muchos de estos grupos y movimientos religiosos se les atribuyen daños tales como distanciamiento respecto a los miembros de la misma familia o amigos, descenso en la escala social por abandono de la formación profesional o del trabajo, dependencia emocional externa frente al grupo o su líder, propensión a la explotación de la fuerza laboral y desde los recursos financieros, pensamiento y lenguaje formalistas y automáticos calcados sobre los esquemas al culto. También argumentó que ocurren situaciones de introversión con predominio del mundo imaginado y desiderativo; temor ante las exigencias del mundo real; desestabilización psíquica en virtud de un desmontaje forzado de los esquemas de orientación habitual y de los necesarios mecanismos de defensa y, como consecuencia, de angustias y depresión, en algunos casos acompañados de agudos episodios psicóticos.

Los estudios de Roberto Assagioli, que une las creencias religiosas con el análisis existencial, demuestran que los trastornos producidos por causas espirituales son actualmente cada vez más numerosos, ya que el número de personas que, consciente o inconscientemente, son constreñidas por exigencias espirituales tam- bién es mayor cada vez. Por este motivo, es oportuno dar una visión general a las alteraciones nerviosas y psíquicas que tienen lugar en los diversos estadios del desarrollo espiritual, y ofrecer algunas indicaciones sobre el modo más apropiado y eficaz para su curación (Assagioli, 1993).

Por otro, lado Pellitero (2009) documenta varios casos de sujetos con estilo de vida religioso que padecían problemas de salud mental causados por adhesión a creencias religiosas particulares y que (contrario a lo que debería pasar), terminó siendo destructivo para las dimensiones personal, laboral y social de dichos sujetos, manifestándose en diversas enfermedades (tanto de origen psíquico como físico). Aun así, estos mismos sujetos obtuvieron tranquilidad y recursos de afrontamiento gracias a la práctica de la oración. Sus síntomas psicóticos lo eran realmente, pues correspondían a una psicosis; pero supieron santificar su enfermedad, hacer de ella un camino hacia Dios. Aunque las consecuencias de su enfermedad podrían calificarse, humana y socialmente, como "destructivas", esas personas, sin embargo, crecieron espiritualmente (Pellitero, 2009, p.524).

Sin embargo, cuando se ejerce el ejercicio clínico, es inevitable la necesidad de muchos pacientes de entender su condición desde la perspectiva de la fe que estos profesan; por ello Rivera-Ledesma \& Montero-López (2007a), consideran que atender estas necesidades debe ser parte fundamental en las buenas prácticas del ejercicio clínico, pues pertenecen a la "realidad clínica cotidiana", especialmente en aquelllas áreas "donde el paciente se ve confrontado con su propia muerte o la de un ser querido" (p.125).

Por tanto, el interés de la presente investigación, gira en torno a la pregunta: ¿Cuál es la relación que existe entre los niveles de salud

Pensamiento Americano Vol. 11 (22) • 2018 • Julio-Diciembre · Corporación Universitaria Americana · Barranquilla, Colombia • ISSN: $2027-2448$. 
mental, derivados de la aplicación del IHM, y las interpretaciones de creencias sobre el carácter de Dios y su confianza en él, de personas pertenecientes a las comunidades de fundamento cristiano, que estudian o laboran en la Corporación Universitaria Adventista y en la Fundación Universitaria Seminario Bíblico de Colombia, de la ciudad de Medellín, durante el año 2017?

Para la OMS (2013), "no hay salud sin salud mental", puesto que para todos los individuos la salud mental, la salud física y la salud social son componentes esenciales de la vida, estrechamente relacionados e interdependientes, (p.6). Se admite, no obstante, que el concepto de salud mental es más amplio que la ausencia de trastornos mentales.

La importancia de la presente investigación se relaciona con la revalidación de la religión en la posmodernidad, no como un mero contenido infaltable en la cultura, sino como un determinante de bienestar y salud mental, un gestor de desarrollo y un proveedor de valores para las sociedades. Por consiguiente, es pertinente a la necesidad que tiene la sociedad de encontrar descripciones objetivas de la religión, ya sea para validarla, revalidarla o simplemente definirla. Por ello, el objetivo de la investigación busca describir a través del IHM (Inventario de Salud Mental) los niveles de salud mental y su posible relación con las interpretaciones de creencias sobre el carácter de Dios y su confianza en él, de personas pertenecientes a comunidades religiosas de fundamento cristiano.

\section{Materiales y métodos}

La presente investigación concibe el estudio de la realidad bajo el paradigma positivista denominado también por Pino (2015) como empírico - analítico, guiada por el enfoque cuantitativo, no experimental. En cuanto al nivel, es descriptiva ya que la recolección de la información se guio fundamentalmente por la observación de fenómenos tal y como se dan en su contexto natural para después analizarlos. Por último, fue transversal, porque se hizo una sola medición en un momento determinado para medir la prevalencia de una exposición o resultado en una población definida y en un punto específico de tiempo. En relación con el ambiente en el cual se desarrolla la investigación, es identificada como "de campo", pues recoge insumos "in situ" en ambientes naturales.

\section{Población y muestra de la investigación}

Los participantes de la investigación fueron miembros regulares de diferentes comunidades religiosas de fundamento cristiano. Como criterio de exclusión se consideró que los miembros de dichas comunidades no ejercieran el liderazgo espiritual en tales iglesias. Se tomó una muestra representativa con miembros de dichas iglesias que en el momento de la recolección de la información se encontraran estudiando y trabajando en la Corporación Universitaria Adventista y en la Fundación Universitaria Seminario Bíblico de Colombia, dado que en ambas instituciones confluye población con las características requeridas para el estudio.

El muestreo probabilístico por racimo sustentado por Hernández, Fernández \& Baptista (2003), fue la técnica para la selección de la muestra; al utilizar el cálculo respectivo se optó por tomar una muestra representativa (estudiantes, docentes y trabajadores de la comunidad educativa cercana a ambas instituciones de educación superior) de 215 sujetos con una prueba piloto previa en la cual participaron 40 personas. Esta fórmula adopta un nivel de confianza del 90\% y un margen de error de $5 \%$.

\section{Recolección de información}

Se utilizó un instrumento con reactivos para las 
dos variables teóricas; la primera parte, indaga el nivel de salud mental; para ello se determinó aplicar las preguntas del Mental Health Inventory (MHI) (Ware, Johnston, Davies-Avery \& Donald, 1979). Este inventario engloba treinta y ocho ítems de respuesta ordinal de cinco o seis puntos que se agrupan en cinco dimensiones, tres negativas y dos positivas. La puntuación total del instrumento resulta del sumatorio de los valores brutos de los ítems que lo componen. Algunos ítems tienen puntuaciones invertidas. Puntuaciones más altas se corresponden con mejor salud mental. Las puntuaciones varían entre 38 y 226 (Fragoeiro, Pestana \& Paúl, 2007). Evalúa la presencia de síntomas durante el último mes con base en una escala de Likert de seis puntos, en un rango de "todo el tiempo" a "nunca". De acuerdo con los autores del instrumento, este permite detectar a la mayoría de las personas que presentan un trastorno según el DSM, con una tasa baja de falsos positivos. El instrumento es de tamizaje y autoaplicable, y aunque no permite concluir un diagnóstico con base en los resultados que arroja. Sin embargo, permite determinar si los sujetos a quienes se aplica presentan síntomas de un probable problema de salud mental (Rodríguez et al., 2008).

Es un cuestionario validado para la población general; evalúan el bienestar y el distrés psicológico en la población general teniendo en cuenta cinco dimensiones: ansiedad; depresión; control conductual/afectivo; afecto positivo general; y emociones. Los resultados del análisis generan dos factores que agrupan los ítems negativos y positivos: distrés psicológico (formado por los negativos); y bienestar psicológico (configurado por los positivos). Este cuestionario puede detectar trastornos del humor y también de ansiedad (Rumpf, Meyer, Hapke \& John, 2001).

Por otro lado, para la variable de "interpretación y confianza en el carácter de Dios", se diseñó un conjunto de reactivos que contienen 14 ítems, también de respuesta ordinal con base en una escala de Likert con cinco opciones de

Tabla 1.

Reactivos para interpretación y confianza en el carácter de Dios

Confía plenamente que cuando se arrepiente de sus pecados Dios lo perdona en forma completa.

Considera que su experiencia religiosa le genera paz interior.

Se siente amado por Dios todo el tiempo independientemente de lo que haga, sea bueno o malo.

Confía plenamente en Dios aun en las pruebas o circunstancias más difíciles de su vida.

Espera pacientemente en Dios aun cuando no tenga respuesta inmediata a sus oraciones.

Tiene la certeza de que Dios responde sus oraciones

Reconoce que Dios cumple sus promesas.

Tiene la confianza en que los que aman a Dios todas las cosas les ayudan a bien.

Está dispuesto a creerle a Dios, aunque no entienda siempre sus caminos.

Su obediencia demuestra una confianza incondicional en Dios.

Siento la tranquilidad de que Dios me acepta tal como soy, es decir, pecador, sin fuerza y necesitado

Vive temeroso de Dios porque Él es castigador.

Cree que Dios aborrece el pecado, pero ama al pecador

Considera que Dios es su Padre celestial y por tanto él es un Padre compasivo.

Fuente: Elaborado por los autores.

Pensamiento Americano Vol. 11 (22) • 2018 • Julio-Diciembre • Corporación Universitaria Americana • Barranquilla, Colombia • ISSN: 2027-2448 · 
respuesta que van desde la descripción "en total desacuerdo", hasta "totalmente de acuerdo". Se encuentran agrupadas en dos dimensiones a saber, "interpretación" y "confianza" en el carácter de Dios, los cuales emergieron, luego del rastreo bibliográfico que desarrollabas ambas dimensiones. Es necesario aclarar que estas dimensiones no se encuentran separadas, sino que integran ambos aspectos. Por ello los reactivos de cada ítem están diseñados de tal forma que permitan visibilizar ambas dimensiones de la variable. La tabla $N^{\circ} 1$ ilustra los reactivos que componen estas dimensiones.

La puntuación total del instrumento resulta del sumatorio de los valores brutos de los ítems que lo componen. Puntuaciones más altas corresponderían con adecuada interpretación y confianza en el carácter de Dios, fundamentado en lo que la Biblia enseña, y en lo que los teóricos del tema sistematizan. Las puntuaciones varían entre 14 y 70 . El objetivo del instrumento es identificar el grado de confianza a partir de la interpretación del carácter de Dios. Por último, el análisis de los datos se llevó a cabo tras la aplicación del cuestionario con el Paquete Es- tadístico para las Ciencias Sociales (SPSS, para Windows), el cual se constituye en un programa modular que implementa gran variedad de temas estadísticos orientados al ámbito de las ciencias sociales.

\section{Resultados}

Al analizar la tabla $N^{\circ} 2$, se puede evidenciar que cuando la población manifiesta tener una "muy alta" confianza en el carácter de Dios, su nivel de salud mental es, "alta", el 31, 2 \% así lo sugiere. En la muestra estudiada hay una incidencia alta entre una adecuada salud mental con relación a una confianza en el carácter de Dios. En la misma línea, se nota que del grupo de sujetos que tienen un alta (o muy alta) confianza en Dios, sus índices de salud mental "baja", representa menos del 1\%, mientras el $15.3 \%$ que presenta una salud moderada tienen una "muy alta" confianza en Dios. Por tanto, es posible deducir que mientras mayor sea la confianza en el carácter de Dios, la tendencia es a tener una buena salud mental en la muestra bajo estudio.

Tabla 2.

Salud mental de acuerdo con la confianza en el carácter de Dios

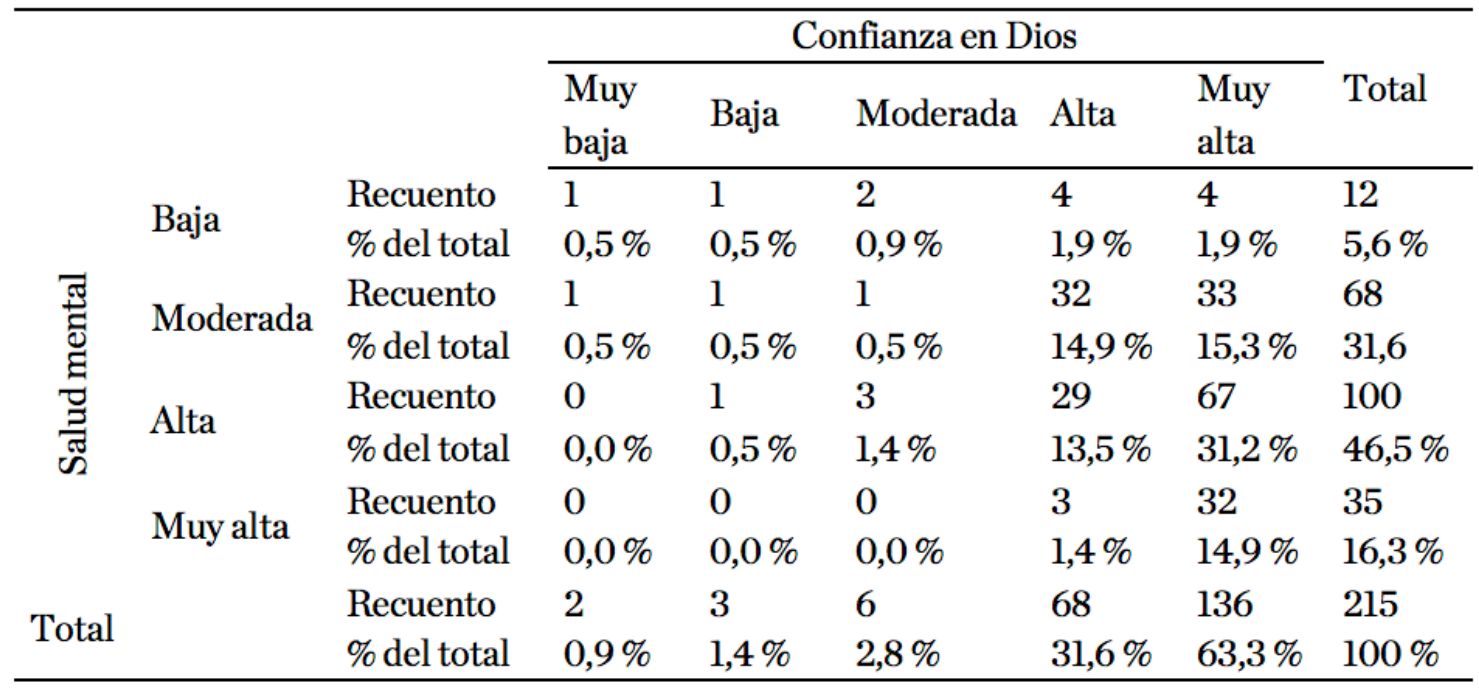

Fuente: Elaborado por los autores. 
La tabla $N^{\circ} 3$ relaciona el nivel de salud mental que presentan miembros de las comunidades religiosas del estudio. De acuerdo con los resultados allí reflejados, se puede observar bajos indicadores de salud mental baja, lo cual confirma el resultado anterior. Es de precisar que la comunidad adventista, es la comunidad con mayor cantidad de sujetos con baja salud mental (con el 8.2\%); en católicos es del $2.1 \%$ y en otras comunidades religiosas es del $4.3 \%$, mientras que el $27,6 \%$ de los adventistas, el 39.6\% de los católicos y 31.9\% de las personas pertenecientes a otras denominaciones presentan una salud mental moderada. En las tres comunidades religiosas estudiadas, se puede observar que un alto porcentaje de sujetos, poseen "alta" salud mental, dado que el $64.3 \%$ de los adventistas, el 58.3\% de los católicos y el $63.8 \%$ de otras denominaciones presentan estas características. Por tanto, se puede observar en la muestra bajo estudio, que en todas las comunidades religiosas existe una tendencia a tener una salud mental adecuada, pero al mismo tiempo, susceptible de mejoramiento.
Como se puede evidenciar en los resultados, la salud mental es fortalecida cuando se tiene confianza en el carácter de Dios. Es por ello importante analizar los índices de confianza en el carácter de Dios de acuerdo con las comunidades religiosas de estudio. La tabla $N^{\circ} 4$ muestra que el porcentaje de baja confianza en el carácter de Dios en la comunidad adventista es del $1.0 \%$, en católicos es del 4.2 $\%$ y ninguna de las personas pertenecientes a otras comunidades religiosas presentaron esta característica, mientras que el $1.0 \%$ de los adventistas, el $6.2 \%$ de los católicos y el $2.9 \%$ de las personas pertenecientes a otras denominaciones presentan una confianza moderada en el carácter de Dios.

En las tres comunidades religiosas estudiadas, se puede observar que un alto porcentaje de las personas de ellas poseen "muy alta" confianza en el carácter de Dios, dado que el 76.5\% de los adventistas, el $43.8 \%$ de los católicos y el 58\% de otras denominaciones presentan estas características. Por tanto, se puede observar en la muestra de estudio, que en todas las

Tabla 3.

Salud mental de las diferentes comunidades religiosas

\begin{tabular}{|c|c|c|c|c|c|}
\hline \multirow{10}{*}{ 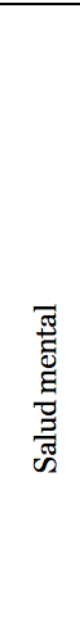 } & & & \multicolumn{3}{|c|}{ Comunidad religiosa } \\
\hline & & & $\begin{array}{l}\text { Adventist } \\
\text { a } \\
\end{array}$ & Católico & $\begin{array}{c}\text { Otras } \\
\text { denominaciones }\end{array}$ \\
\hline & & Recuento & 8 & 1 & 3 \\
\hline & Baja & $\begin{array}{l}\% \text { dentro de comunidad } \\
\text { religiosa }\end{array}$ & $8,2 \%$ & $2,1 \%$ & $4,3 \%$ \\
\hline & & Recuento & 27 & 19 & 22 \\
\hline & Moderada & $\begin{array}{l}\% \text { dentro de comunidad } \\
\text { religiosa }\end{array}$ & $27,6 \%$ & $39,6 \%$ & $31,9 \%$ \\
\hline & & Recuento & 42 & 22 & 36 \\
\hline & Alta & $\begin{array}{l}\% \text { dentro de comunidad } \\
\text { religiosa }\end{array}$ & $42,9 \%$ & $45,8 \%$ & $52,2 \%$ \\
\hline & & Recuento & 21 & 6 & 8 \\
\hline & Muy alta & $\begin{array}{l}\% \text { dentro de comunidad } \\
\text { religiosa }\end{array}$ & $21,4 \%$ & $12,5 \%$ & $11,6 \%$ \\
\hline & & Recuento & 98 & 48 & 69 \\
\hline Total & & $\begin{array}{l}\% \text { dentro de comunidad } \\
\text { religiosa }\end{array}$ & $100 \%$ & $100 \%$ & $100 \%$ \\
\hline
\end{tabular}

Fuente: Elaborado por los autores. 
comunidades religiosas existe una tendencia a tener una confianza en Dios muy alta.

La tabla $\mathrm{N}^{\circ} 5$ analiza el nivel de confianza en el carácter de Dios de acuerdo con el tiempo en el cual, el sujeto pertenece a dicha iglesia. Allí se refleja una tendencia a tener una "alta" confianza en el carácter de Dios independientemente de los años que las personas lleven en la iglesia. La misma conclusión si se piensa en la tabla 6; es posible deducir de acuerdo con la muestra que existe una tendencia a tener una alta confianza en el carácter de Dios independientemente de los años que las personas lleven en la iglesia.

Por último, considerando las siguientes hipótesis:

$\mathrm{H}_{0}$ : No existe relación entre los niveles de salud mental, y la confianza en el carácter de Dios, de personas pertenecientes a las comunidades de fundamento cristiano, que estudian o laboran en la Corporación Universitaria Adventista y en la Fundación Universitaria Seminario Bíblico de Colombia, de la ciudad de Medellín, durante el año 2017.

Ha: Existe relación entre los niveles de salud mental, y la confianza en el carácter de Dios, de personas pertenecientes a las comunidades de fundamento cristiano, que estudian o laboran en la Corporación Universitaria Adventista y en la Fundación Universitaria Seminario Bíblico de Colombia, de la ciudad de Medellín, durante el año 2017.

La tabla 7 muestra que como el valor-p es pequeño (Valor $p<0.001$ ), hay evidencia para decir que existe relación entre los niveles de salud mental, y la confianza en el carácter de Dios. Con base en el coeficiente de correlación rho

Tabla 4

Confianza en Dios de las diferentes comunidades religiosas

\begin{tabular}{|c|c|c|c|c|c|}
\hline & & & \multicolumn{3}{|c|}{ Comunidad religiosa } \\
\hline & & & Adventista & Católico & $\begin{array}{c}\text { Otras } \\
\text { denominacione } \\
\mathbf{s} \\
\end{array}$ \\
\hline & & Recuento & 0 & 2 & 0 \\
\hline & Muy baja & $\begin{array}{l}\% \text { dentro de } \\
\text { comunidad religiosa }\end{array}$ & $0,0 \%$ & $4,2 \%$ & $0,0 \%$ \\
\hline & & Recuento & 1 & 2 & 0 \\
\hline & Baja & $\begin{array}{l}\% \text { dentro de } \\
\text { comunidad religiosa }\end{array}$ & $1,0 \%$ & $4,2 \%$ & $0,0 \%$ \\
\hline & & Recuento & 1 & 3 & 2 \\
\hline \multirow{6}{*}{$\begin{array}{l}\text { Confianza } \\
\text { en Dios }\end{array}$} & Moderada & $\begin{array}{l}\% \text { dentro de } \\
\text { comunidad religiosa }\end{array}$ & $1,0 \%$ & $6,2 \%$ & $2,9 \%$ \\
\hline & & Recuento & 21 & 20 & 27 \\
\hline & Alta & $\begin{array}{l}\% \text { dentro de } \\
\text { comunidad religiosa }\end{array}$ & $21,4 \%$ & $41,7 \%$ & $39,1 \%$ \\
\hline & & Recuento & 75 & 21 & 40 \\
\hline & Muy alta & $\begin{array}{l}\% \text { dentro de } \\
\text { comunidad religiosa }\end{array}$ & $76,5 \%$ & $43,8 \%$ & $58 \%$ \\
\hline & & Recuento & 98 & 48 & 69 \\
\hline Total & & $\begin{array}{l}\% \text { dentro de } \\
\text { comunidad religiosa }\end{array}$ & $100 \%$ & $100 \%$ & $100 \%$ \\
\hline
\end{tabular}

Fuente: Elaborado por los autores. 
Tabla 5.

Confianza en Dios de acuerdo con el tiempo en la iglesia.

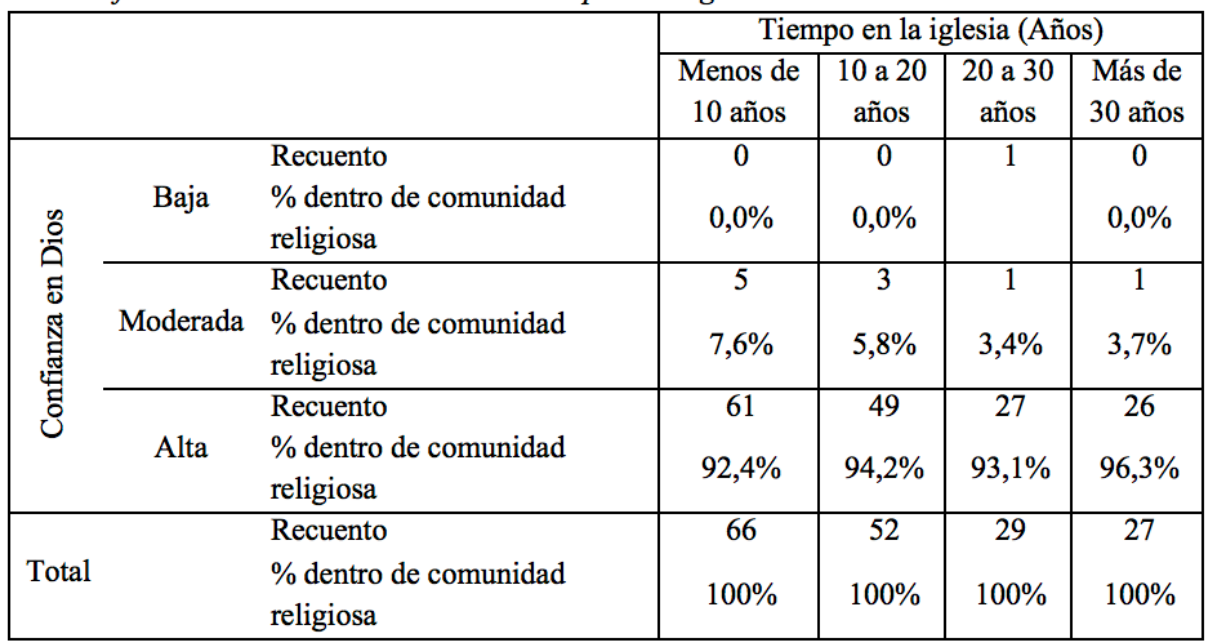

Fuente: Elaborado por los autores

Tabla 6.

Salud mental de acuerdo con el tiempo en la iglesia

\begin{tabular}{|c|c|c|c|c|c|c|}
\hline & \multicolumn{4}{|c|}{ Tiempo en la iglesia (Años) } \\
\hline & & & $\begin{array}{l}\text { Menos de } 10 \\
\text { años }\end{array}$ & $\begin{array}{c}10 \text { a } 20 \\
\text { años }\end{array}$ & $\begin{array}{c}20 \text { a } 30 \\
\text { años }\end{array}$ & $\begin{array}{l}\text { Más de } \\
30 \text { años }\end{array}$ \\
\hline \multirow{6}{*}{$\begin{array}{c}\text { Salud } \\
\text { mental }\end{array}$} & \multirow{3}{*}{ Baja } & Recuento & 3 & 5 & 3 & 0 \\
\hline & & $\begin{array}{l}\% \text { dentro de comunidad } \\
\text { religiosa }\end{array}$ & $4,5 \%$ & $9,6 \%$ & $10,3 \%$ & $0,0 \%$ \\
\hline & & Recuento & 22 & 16 & 8 & 6 \\
\hline & \multirow[t]{2}{*}{ Moderada } & $\begin{array}{l}\% \text { dentro de comunidad } \\
\text { religiosa }\end{array}$ & $33,3 \%$ & $30,8 \%$ & $27,6 \%$ & $22,2 \%$ \\
\hline & & Recuento & 41 & 31 & 18 & 21 \\
\hline & \multirow[t]{2}{*}{ Alta } & $\begin{array}{l}\% \text { dentro de comunidad } \\
\text { religiosa }\end{array}$ & $62,1 \%$ & $59,6 \%$ & $62,1 \%$ & $77,8 \%$ \\
\hline \multirow[b]{2}{*}{ Total } & & Recuento & 66 & 52 & 29 & 27 \\
\hline & & $\begin{array}{l}\% \text { dentro de comunidad } \\
\text { religiosa }\end{array}$ & $100 \%$ & $100 \%$ & $100 \%$ & $100 \%$ \\
\hline
\end{tabular}

Fuente: Elaborado por los autores.

Tabla 7.

Correlación.

\begin{tabular}{ccccc}
\hline & & $\begin{array}{c}\text { Confianza en } \\
\text { Dios }\end{array}$ & $\begin{array}{c}\text { Salud } \\
\text { mental }\end{array}$ \\
\cline { 3 - 5 } & & & 1,000 &, 337 \\
Rho de & Confianza & Coeficiente de correlación & &, 000 \\
Spearman & & Sig. (bilateral) & & 215 \\
& & N & 215 & 1,000 \\
& Salud & Coeficiente de correlación &, 337 & \\
& mental & Sig. (bilateral) &, 000 & \\
& & $\mathrm{~N}$ & 215 & 215 \\
\hline
\end{tabular}

Fuente: Elaborado por los autores. 
de Spearman de $\rho=0.337$, se puede deducir que el grado de correlación entre los niveles de salud mental, y la confianza en el carácter de Dios. Por ende, los niveles de salud mental tienen una posible relación con las interpretaciones de creencias sobre el carácter de Dios y su confianza en él, de personas pertenecientes a comunidades religiosas de fundamento cristiano.

\section{Discusión}

Las comunidades religiosas de fundamento cristiano centran su estilo de vida, a partir de la convicción de la vida de Jesús, como personaje histórico, fundador y guía de fe, que tiene poder providencial para gobernar, no solo el universo físico y tangible, o el espiritual e intangible, sino, las diferentes dimensiones del ser humano. Sin embargo, al introducir el concepto de libre albedrío, en la individualidad del sujeto, al interior de sus comunidades se visibilizan escenarios en los que interaccionan sujetos que padecen dificultades mentales, psicopatológicas, individuales o sociales; $y$, al mismo tiempo, son ambientes terapéuticos que evitan que los individuos caigan en disfunciones mentales, o ayudan a que se sobrepongan a ellas, lo que revalida la cuestión sobre la práctica religiosa en la posmodernidad, no como un mero contenido infaltable en la cultura, sino como un determinante de bienestar y salud mental, un gestor de desarrollo y un proveedor de valores para las sociedades.

En este sentido, y considerando la relación dialéctica entre la teología y la psicología, la presente investigación toma como marco de referencia la fenomenología. Desde allí, se hace necesario continuar investigando sobre salud mental y religiosidad, pues, observar la religión, y en especial la salud mental dentro de la religión, es observar la dinámica psicosocial de esta región. Por ejemplo, la psicología positiva afirma que la religiosidad no debe conside- rarse como anormal o un problema de salud mental; por el contrario, hace una conexión de la religiosidad con el bienestar de las personas en las diferentes áreas de la vida (Rosales \& Schinelli, 2011).

Desde esta perspectiva quedan sustentados los resultados de la presente investigación que muestran una relación entre los niveles de salud mental y las interpretaciones de creencias sobre el carácter de Dios y su confianza en él, de personas pertenecientes a comunidades religiosas de fundamento cristiano, y que validan las propuestas de otras producciones como las de Rivera-Ledesma y Montero-López (2005; 2007b); Jaramillo-Vélez, Ospina-Muñoz, Cabarcas-Iglesias y Janice Humphreys (2005); Rodríguez (2011); Navas y Villegas (2006); Espíndula, Valle y Bello (2010); Quiceno \& Vinaccia (2009); Valiente-Barroso \& García-García (2010).

Un aspecto por considerar sobre esta relación, y que se vio reflejado en los resultados de la presente investigación, es la posición de Rivera-Ledesma \& Montero-López (2005), quienes refieren que "orar, leer la Biblia, pronunciar expresiones de confianza y fe en Dios" afectan positivamente la salud y propician sentido de bienestar personal (p. 53). Este argumento lo amplían Valiente-Barroso \& García-García (2010), quienes, citando a Levin (2009), explican un enfoque explicativo biopsicosocial, caracterizado por la dimensión conductual, en donde "la fe poseería la capacidad de condicionar y regular directamente los sistemas endocrino e inmunológico"; la dimensión interpersonal, que proporciona al creyente el desarrollo de la empatía, la cual le permitiría establecer adecuadas interacciones; la dimensión cognitiva, que establecería en el creyente "una estructura mental que configura una aptitud para un adecuado afrontamiento en lo relativo al dolor, el sufrimiento y otros componentes asociados a las patologías"; la dimensión afectiva, toda vez que "los sentimientos positivos elicitados

Pensamiento Americano Vol. 11 (22) · 2018 • Julio-Diciembre · Corporación Universitaria Americana • Barranquilla, Colombia • ISSN: $2027-2448$. 
a través de pensamientos, creencias y experiencias ... basados en la fe pueden influir sobre el impacto físico que pueden producir algunas entidades clínicas, así como modular parámetros del sistema inmune indicativos de fisiopatología"; y por último, la dimensión psicofisiológica, en la cual "la fe proporcionaría optimismo y esperanza en el futuro, ayudando a que situaciones generadoras de dolor y malestar puedan tolerarse mejor; su efecto mental en el individuo se ha constatado perfectamente". La creencia y la confianza, asociadas a la fe religiosa, están en consonancia con ese tipo de acciones mentales conocidas por producir respuestas corporales cuantificables y clínicamente significativas (Levin 2009, citado en Valiente-Barroso \& García-García, 2010, p.158).

En lo que respecta al carácter de Dios, es importante referirla como lo haría Méndez (1994) quien lo relaciona con la justicia, asociándolo con un rey y juez, quienes "están llamados a rescatar a los oprimidos y desvalidos (Sal. 72:14; 82:1-18). El hacerlo equivale a participar en los hechos salvífícos de Dios, quien preserva a su pueblo de los efectos del pecado (Sal. 48:12-18; 140:12ss; 146:7-10; Is. 1:17; Jer 22:16)". (p.133). Y, en cuanto a la confianza, fundamento sustentado en los resultados del presente estudio, es necesario establecer que la misma, "es un acto humano que referimos a personas, por tanto, a sujetos libres". Cuando la confianza es dirigida hacia Dios, Spaemann (2010) considera que es "absoluta e inquebrantable ocurra lo que ocurra... la fe de que en el futuro nos irá, si no mejor, por lo menos no sustancialmente peor que hasta ahora" (p. 137).

Llama la atención que Spaemann relaciona el concepto de confianza con "libertad", término imprescindible para la comunidad religiosa de carácter cristiano, quienes además, interpretan que, más que un deber moral, la conEl énfasis es de los autores

Énfasis de los autores fianza es una invitación por parte de Dios y se evidencia en textos como "Venid a mí todos los que estáis trabajados y cargados, y yo os haré descansar"; Jeremías 39:18: "Porque ciertamente te libraré, y no caerás a espada, sino que tu vida te será por botín, porque tuviste CONFIANZA' en mí, dice Jehová"; Juan 14:27: "La paz os dejo, MI PA ${ }^{2} Z$ os doy; yo no os la doy como el mundo la da. No se turbe vuestro corazón, ni tenga miedo"; Jeremías 31:25: "Porque satisfaré al alma cansada, y saciaré a toda alma entristecida" (Reina-Valera, 1960).

El antropólogo Manuel Marzal (2004, p.20), especialista en la religiosidad popular peruana y en general latinoamericana, resume sus investigaciones en el modo siguiente: "La clave de la religión del pueblo está en el significado que el pueblo latinoamericano da a ocho palabras ("devoción", "santo", "milagro", "castigo", "promesa", "bendición", "fiesta" y "peregrinación) (citado en Lecaros, 2017, p.572). Todas estas expresiones, relacionadas por Marzal, ilustran algunos descriptores de confianza en el carácter de Dios. Ya que la libertad que se encuentra implícita en la confianza en el carácter de Dios realmente es una percepción cognitiva de la realidad subjetiva del sujeto que lo lleva a adquirir actitudes, sentimientos y comportamientos, supeditados por esta representación. Al respecto de esta percepción, el autor plantea una paradoja (que a continuación se relaciona) que refleja la diversidad en dichas representaciones:

En el imaginario culturalmente enraizado en las tradiciones, dominan percepciones divinas como de un juez temible, todo poderoso, ordenador del cosmos y eventualmente castigador del malvado; por otro lado, se expresa el cariño a un Dios protector y proveedor. Ambas perspectivas se armonizan y se complementan en un Dios garante del orden justo del mundo. En contexto evangélico, la concepción de Dios no 
varía mucho, se exacerba la importancia del poder divino (Lecaros, 2017, p.571).

Estas concepciones del carácter Dios presentan matices que estructuran el pensamiento religioso del individuo, llevándolo a una experiencia religiosa particular que lo traslada tanto a una vida tranquila, y que evidencia la expresión bíblica de Jesús: "Mi paz os dejo, mi paz os doy", o le termina generando, como ya fue mencionado, desestabilización psíquica en virtud de un desmontaje forzado de los esquemas de orientación habitual y de los necesarios mecanismos de defensa, y como consecuencia de angustias y depresión, en algunos casos acompañados de agudos episodios psicóticos.

\section{Conclusión}

Como ya se mencionó, el presente estudio concluye que existe relación entre los niveles de salud mental y las interpretaciones de creencias sobre el carácter de Dios y su confianza en él, de personas pertenecientes a comunidades religiosas de fundamento cristiano, y, a pesar que, aún existe un gran espectro de interrogantes, posibilidades y retos que se ciernen sobre este estudio, se avanza en una línea empírica de descripción de realidades que permiten visibilizar algunas concepciones teóricas de estudios que la preceden. Lo importante ahora es entender que más allá del reconocimiento de la importancia de este tema para la comprensión de las dinámicas sociales, es necesario ahondar en ellas, para comprender la complejidad del fenómeno religioso en la vida de los seres humanos.

\section{Referencias}

Assagioli, R. (1993). Psicosíntesis: Ser transpersonal: el nacimiento de nuestro ser real. Móstoles, Madrid: Gaia Ediciones.

Cordelia, B. \& Kirsten, A. F. (2010). Intersections of Gender, Religion and Ethnicity in the Middle Ages. Palgrave Macmillan, Basingstoke, GB.

Revelan radiografía de la salud mental en Medellín. (7 de enero de 2016). El Tiempo. Recuperado de: http://www.eltiempo.com/archivo/documento/CMS16474692

Hay que invertir más en salud mental: OMS. (12 de octubre de 2011). El Universal. Recuperado de: https:// www.eluniversal.com.co/salud/hay-que-invertirmas-en-salud-mental-oms-48061-NTEU128432

Espíndula, J., Valle, E. \& Bello, A. (2010). Religión y espiritualidad: una perspectiva de profesionales de la salud. Revista Latino-Americana de Enfermagem, 18(6), 1229-1236. https://dx.doi.org/10.1590/s010411692010000600025

Fragoeiro, I., Pestana, M. H. \& Paúl, C. (2007). La salud mental en los ancianos de la Región Autónoma de Madeira: Estudio piloto. Gerokomos, 18(4), 23-27.

Hauck, R. J. (1985). Inspiration as apologetic: The debate over true prophecy in the Contra Celsum of Origen. Duke University.

Hernández Sampieri, R., Fernández Collado, C. \& Baptista Lucio, P. (1991). Metodología de la investigación (Segunda ed.). México: McGraw-Hill.

Jaramillo-Vélez, D. E., Ospina-Muñoz, D., Cabarcas-Iglesias, G. \& Humphreys, J. (2005). Resiliencia, espiritualidad, aflicción y tácticas de resolución de conflictos en mujeres maltratadas. Rev Salud Pública, 7, 281292.

Lecaros, V. (2017). ¿Dios castigador, Dios juez o Dios amado? Imágenes de Dios en medios católicos y pentecostales peruanos. Horizonte, 15(46), 571-594. doi: http://dx.doi.org/10.5752/P.2175-5841.2017v15n46p571

Méndez, G. W. (1994). La justicia es social si refleja el carácter de Dios. Guatemala: Kairós. 119-139.

Navas, C. \& Villegas, H. (2006). Espiritualidad y salud. Re- 
vista Ciencias de la Educación, 1(27), 29-45.

OMS. (2004). http://mww.who.int/. Recuperado el 27 de abril de 27, de http://www.who.int/mental_health/ advocacy/en/spanish_final.pdf

OMS. (diciembre de 2013). Organización Mundial de la Salud. Obtenido de Salud mental: un estado de bienestar: http://www.who.int/features/factfiles/ mental_health/es/

Oramas Viera, A., Santana López, S. \& Vergara Barrenechea, A. (2006). El bienestar psicológico, un indicador positivo de la salud mental. Revista Cubana de Salud y Trabajo, 7(1-2). 34 - 39.

Pellitero, R. (2009). Religion and psychiatry: routes that converge. Scriptatheologica, 41(2), 511-538.

Quiceno, J. M. \& Vinaccia, S. (2009). La salud en el marco de la psicología de la religión y la espiritualidad. Revista Diversitas, 5(2), 321-336.

Ramos Lorente, M. (2006). Nuevos movimientos religiosos en España: contexto y análisis del proceso de afiliación y desvinculación de sus miembros. Tesis doctoral. Universidad de Granada. España.

Rivera-Ledesma, A. \& Montero-López, L. M. (2005). Espiritualidad y religiosidad en adultos mayores mexicanos. Salud Mental, 28(6), 51-58.

Rivera-Ledesma, A. \& Montero-López, L. M. (2007a). Ejercicio clínico y espiritualidad. Anales de Psicología 23(1). $125-136$

Rivera-Ledesma, A. \& Montero-López, L. M. (2007b). Medidas de afrontamiento religioso y espiritualidad en adultos mayores mexicanos. Salud Mental, 30(1) 39-47.

Rodríguez, M. A., Martínez, V. N., Juárez, G. F., López, L. E., Carreño, G. S. \& Medina-Mora, M. E. (2008). Relación entre el consumo de tabaco, salud mental y malestares físicos en hombres trabajadores de una empresa textil mexicana. Salud Mental, 31(4). 291 - 297.

Rodríguez, N. (2011). Impacto de la espiritualidad en salud mental. Una propuesta de estrategia de atención comunitaria de salud mental en colaboración con grupos religiosos locales. Rev. GPU, 7(2), 205-213.

Rosales, M. G. \& Schinelli, F. (2011). Religión y salud men- tal: diagnóstico, investigaciones y tratamientos. III Congreso Internacional de Investigación y Práctica Profesional en Psicología XVIII. Jornadas de Investigación Séptimo Encuentro de Investigadores en Psicología del MERCOSUR (pág. 5). Buenos Aires.: Universidad de Buenos Aires.

Rumpf, H. J., Meyer, C., Hapke, U. \& John, U. (2001). Screening for mental health: validity of the MHI-5 using DSM-IV Axis I psychiatric disorders as gold standard. Psychiatry Research, 105(3), 243-253.

Santa Biblia. Versión de Casiodoro Reina (1960) Revisada por Cipriano Valera (1602) -Revisión 1960. Philadelphia, Pensilvania, USA: Sociedades Bíblicas en America Latina, National Publishing Company.

Suárez Rueda. M. (2010). La salud mental de los colombianos, en crisis. El Espectador. Recuperado de: https://www.elespectador.com/noticias/salud/articulo-234929-salud-mental-de-los-colombianos-crisis

Spaemann, R. (2010). Confianza. Recuperado de: http://dadun.unav.edu/bitstream/10171/7007/4/Confianza.pdf

Pino Montoya, J. W. (2015). Metodología de la investigación en la ciencia política: La mirada empírico - analítica. Revista Fundación Universitaria Luis Amigó. 2(2). 185-195.

Valiente-Barroso, C. \& García-García, E. (2010). La religiosidad como factor promotor de salud y bienestar para un modelo multidisciplinar de atención psicogeriátrica. Psicogeriatría, 2(3), 153-165.

Ware, J., Johnston, S., Davies-Avery, A. \& Donald, C. (1979). Conceptualization and measurement of health for adults in the Health Insurance Experiment. Vol III: Mental Health. e-HEW. The Rand Corporation, Santa Monica.

2018, Vol. 11(22) 121-134 @The Author(s) 2018 Reprints and permission: www.americana.edu.co

https://www.coruniamericana.edu.co/publicaciones/ojs/index.php/pensamientoamericano/index 